\title{
L'introduction de l'identification systématique pour réduire les besoins non satisfaits en santé : Manuel du manager
}

Ricardo Vernon

James R. Foreit

Population Council

Emma Ottolenghi

Follow this and additional works at: https://knowledgecommons.popcouncil.org/departments_sbsr-rh

Part of the Demography, Population, and Ecology Commons, Family, Life Course, and Society Commons, International Public Health Commons, and the Medicine and Health Commons How does access to this work benefit you? Let us know!

\section{Recommended Citation}

Vernon, Ricardo, James R. Foreit, and Emma Ottolenghi. 2008. "L'introduction de l'identification systématique pour réduire les besoins non satisfaits en santé : Manuel du manager," FRONTIERS Manual. Washington, DC: Population Council. 


\section{L'introduction de l'identification systématique pour réduire les besoins non satisfaits en santé : Manuel du manager}

Ricardo Vernon

James R. Foreit

Emma Ottolenghi
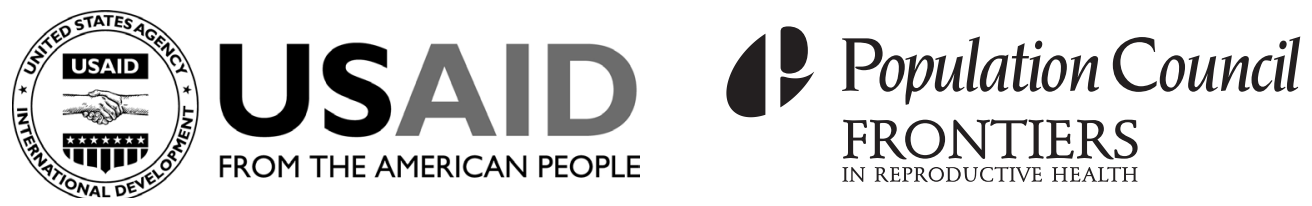

Cette publication a été possible grâce au soutien fourni par l'AGENCE DES ETATS-UNIS POUR LE DEVELOPPEMENT INTERNATIONAL (USAID) aux termes de l'Accord collaboratif numéro HRN-A-00-98-00012-00. Les opinions exprimées ici incombent aux auteurs et ne reflètent pas forcément les vues de l'USAID. 


\section{Population Council}

Le Population Council est une institution internationale, non gouvernementale et à but non lucratif dont la mission est d'améliorer le bien-être et la santé de la reproduction de la génération actuelle et de celle à venir dans le monde et d'arriver à un équilibre humain équitable et soutenable entre les êtres et les ressources. Le Council réalise une recherche biomédicale, en sciences sociales et en santé publique et aide à renforcer les capacités en recherche dans les pays en développement. Fondé en 1952, le Council est régi par un conseil d'administration international. Son siège à New York vient soutenir un réseau mondial de bureaux regionaux et nationaux.

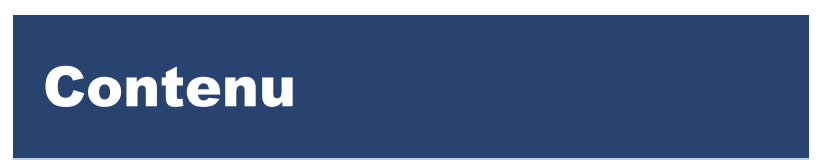

Ce manuel

Comment appliquer

l'identification systématique

Références

Annexe:

Exemple de plan de formation

\section{Frontiers in Reproductive Health}

Frontiers in Reproductive Health Program (FRONTIERS) applique des techniques de recherche systématique pour améliorer la prestation des services de planification familiale et de santé de la reproduction et pour influencer les politiques connexes. FRONTIERS est financé par l'Agence des Etats-Unis pour le Développement international (USAID) et est mis en œuvre par le Population Council en collaboration avec Family Health International. Le personnel de FRONTIERS et les organismes collaborateurs réalisent une recherche opérationnelle en Afrique, en Asie et au Proche-Orient, en Europe de l'Est, en Amérique latine et aux Caraïbes.

Frontiers in Reproductive Health Program (FRONTIERS)

Population Council

4301 Connecticut Ave., NW, Suite 280

Washington, DC 20008 USA

Téléphone : (202) 237-9400

Fax : (202) 237-8410

Courriel : publications@popcouncil.org

www.popcouncil.org/frontiers

Cette publication a été possible grâce au soutien fourni par I'AGENCE DES ETATS-UNIS POUR LE DEVELOPPEMENT INTERNATIONAL (USAID) aux termes de l'Accord collaboratif numéro HRN-A-00-98-00012-00. Les opinions exprimées ici incombent aux auteurs et ne reflètent pas forcément les vues de I'USAID.

\section{Révisé 2008}

(c) 2006 Population Council, Inc.

Cette publication peut être reproduite partiellement ou intégralement sans l'autorisation du Population Council du moment qu'est mentionnée la source complète et que la reproduction ne sert pas à des fins commerciales.

Titre original: Introducing Systematic Screening to Reduce Unmet Health Needs: A Manager's Manual.

Citation proposée : Vernon, Ricardo, James R. Foreit et Emma Ottolenghi. 2008. " L'introduction de l'identification systématique pour réduire les besoins non satisfaits en santé : Manuel du manager, "FRONTIERS Manual, édition révisée. Washington, DC: Population Council. 


\section{Ce manuel}

Ce manuel vise à aider les décideurs de programme, les responsables, les superviseurs et les prestataires de soins à introduire l'identification systématique dans leurs services de santé. Cette identification systématique peut améliorer la santé des femmes en répondant aux multiples besoins non satisfaits en services de santé de la reproduction et autres services de santé. Ce manuel présente l'information et les outils nécessaires pour ajouter l'identification systématique à un programme de santé :

Activités nécessaires pour mettre en place l'identification systématique.

- Comment choisir les centres de santé et les services qui feront l'objet d'une identification ainsi que les personnes qui seront dépistées.

- Mise au point, adaptation et prétest avec exemples d'une liste de vérification de l'identification systématique.

- Conseils pour la formation des agents d'identification/prestataires et superviseurs et programme de formation générique.

Le contenu du manuel peut être adapté pour répondre aux priorités et ressources en santé, aux niveaux local, national ou programmatique, ainsi que pour promouvoir l'utilisation des services préventifs sousutilisés.

\section{Le problème : Besoin non satisfait de services de santé de la repro- duction}

La plupart des femmes qui viennent consulter les services de santé ont de nombreux besoins sur le plan santé de la reproduction et santé infantile, tant préventifs que curatifs. Il peut s'agir de consultations prénatales et du post-partum, de la planification familiale, du suivi-croissance de l'enfant et de la vaccination, des bilans de santé et du suivi nutritionnel, des services préventifs et de l'identification du cancer du col, et de l'identification et traitement des infections sexuellement transmissibles et VIH. Géné- ralement, les prestataires ne dispensent que le service demandé par les clientes et celles-ci ne savent pas toujours que leur santé pourrait bénéficier de services supplémentaires ou que les services nécessaires sont disponibles. Aussi, la cliente risque-t-elle de quitter l'établissement avec des besoins non satisfaits en santé de la reproduction et le prestataire de soins rate-t-il l'occasion de lui dispenser ses services.

\section{Une solution : Intégration des ser- vices de la santé de la reproduc- tion au niveau du prestataire}

Le Programme d'Action de la Conférence internationale sur la Population et le Développement (CIPD) du Caire, en 1994, recommande des services intégrés de santé de la reproduction. Par intégration, on entend prestation proactive de multiples services de santé de la reproduction dans le même établissement au même moment (Foreit, Hardee, et Agarwal 2002). A cette fin, il est nécessaire de définir les besoins et souhaits de la cliente sur le plan de la santé de la reproduction quand elle arrive dans l'établissement puis de lui fournir ses services pendant la même visite, lors d'un rendez-vous ultérieur ou en l'orientant vers un autre établissement.

\section{Identification systématique : Une technique simple pour une inté- gration efficace}

L'identification systématique a été conçu comme une des « meilleures pratiques" par l'USAID. La technique comprend trois étapes :

1. Utiliser un instrument standardisé (ensemble de questions) pour déterminer les besoins de chaque cliente pour des services supplémentaire en santé de la reproduction et santé infantile.

2. S'ils sont disponibles, dispenser les services identi fiés pendant la même visite ; ou

3. Offrir un autre rendez-vous ou l'orienter vers les services qui ne peuvent pas être fournis immédiatement. 


\section{Donnée probante : L’identification systématique est une formule réussie}

- Les besoins non satisfaits de la cliente diminuent. Au Mexique, le taux de vaccination a augmenté, passant de $4 \%$ à $33 \%$ parmi les enfants qui n'étaient pas venus au centre de santé pour ce service (Vernon et Foreit 1999).

- Le nombre de services par visite augmente une fois qu'ils sont introduits dans le programme l'identification systématique. Dans le cadre d'études faites en Afrique, en Asie et en Amérique latine, l'identification systématique a permis d'augmenter les services par visite, de l'ordre de $9 \%$ à $28 \%$, suivant l'établissement. Les accroissements notables dans l'utilisation de services et la comparabilité de ces résultats, montrés sur le Tableau 1 ci-après, montrent bien que l'identification systématique s'avère efficace dans différents pays, programmes et contextes.

- La plupart des besoins non satisfaits identifiés entraînent une prestation de services supplémentaires. En Inde, 96\% ont abouti à des services supplémentaires, surtout en planification familiale. Les services effectivement dispensés étaient différents selon le contexte et la stratégie d'identification.

- Une seule visite plutôt que de multiples visites pour les services nécessaires diminue les frais et le temps des clientes.

- L'accroissement dans le nombre de services fournis par visite suppose une amélioration dans la productivité des prestataires (voir Brambila et Solórzano 1998). Dans les régions où il existe un besoin non satisfait élevé pour des services de santé de la reproduction et où la productivité des prestataires de soins est faible, l'identification est une autre possibilité efficace par rapport aux coûts que la stratégie avancée plus onéreuse.

- Les établissements de santé peuvent obtenir davantage de recettes grâce aux services supplémentaires fournis. Un petit programme clinique au Pérou a obtenu des recettes supplémentaires d'environ 42000 \$US par an en utilisant l'identification systématique (León et al. 1998).
Vu que l'outil a effectivement permis d'accroître le nombre de services prioritaires fournis, l'identification systématique a été intégré à des programmes au Guatemala, en Inde et au Sénégal et est utilisé pour dépister un grand nombre de besoins différents sur le plan des services.

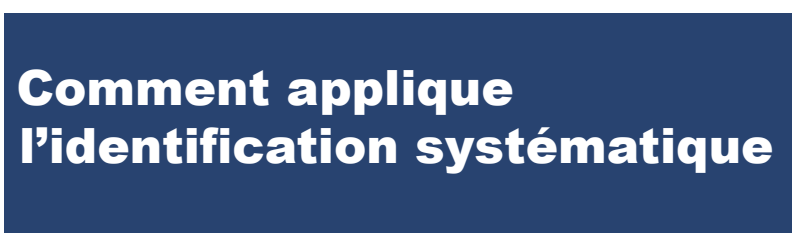

Sont décrites ci-après les activités essentielles pour mettre à l'œuvre la stratégie de l'identification systématique :

\section{Confier l'identification à une personne qui en sera responsable}

Souvent, une innovation n'aboutit pas car il n'existe pas une personne qui en est responsable-quelqu'un qui doit vérifier que l'innovation est effectivement appliquée comme prévu. Il est important de nommer un responsable de votre programme d'identification en général et un responsable du programme dans chaque établissement.

Au niveau du programme le coordinateur est responsable de mettre au point la fiche d'identification, d'organiser l'identification, de former le personnel, de superviser l'intervention et de rendre compte des résultats de l'identification aux responsables/décideurs du programme.

Au niveau de l'établissement une personne doit être responsable de vérifier que les fiches d'identification sont effectivement disponibles, que le personnel actuel et nouveau a été formé pour appliquer la technique et que les prestataires font effectivement l'identification des clientes. Un superviseur devrait être le responsable lorsqu'il existe de multiples petits postes avec un seul prestataire.

\section{Décider du champ d'action de l'identification}

- Faire une identification pour seuls quelques services prioritaires pour lesquels il existe un important besoin non satisfait. Il vaut mieux faire une identification 
des services comportant de nets avantages pour la santé et pour lesquels il est possible de dispenser les services en tout temps (c'est-à-dire, pour lesquels il existe les fournitures et le personnel formé) tout en vérifiant que ces services sont également sousutilisés (c'est-à-dire, pour lesquels il existe une faible demande et une capacité excédentaire de prestation de services).

- Ne pas faire d'identification pour un trop grand nombre de besoins de service. La procédure prend trop longtemps, devient trop compliquée et décourage le personnel. De longs entretiens peuvent également ennuyer les clientes. Et en plus, si on identifie un nombre important de besoins de service, la femme risque de n'avoir ni le temps ni les ressources pour les recevoir tous. La fiche présentée ici est conçue pour des services à l'intention de femmes et d'enfants, mais pourrait être adaptée également pour des services destinés aux hommes.

\section{Tableau 1.}

\section{Efficacité de l'identification systématique pour augmenter les services par visite: femmes non dépistées contre femmes dépistées}

\begin{tabular}{lccc|}
\hline Pays & $\begin{array}{c}\text { Services } \\
\text { par visite : } \\
\text { femmes } \\
\text { non dépis- } \\
\text { tées }\end{array}$ & $\begin{array}{c}\text { Services } \\
\text { par visite : } \\
\text { femmes } \\
\text { dépistées }\end{array}$ & $\begin{array}{c}\text { Différence } \\
(\%)\end{array}$ \\
\hline Bolivie & 1,1 & 1,2 & 9 \\
\hline Honduras & 1,1 & 1,3 & 18 \\
\hline Pérou & 1,6 & 1,8 & 13 \\
\hline Inde & 1,6 & & \\
$\begin{array}{l}\text { (établissements } \\
\text { plus grands) } \\
\text { (petits postes) }\end{array}$ & 1,5 & 1,6 & 7 \\
\hline $\begin{array}{l}\text { Sénégal } \\
\text { (centres } \\
\text { urbains) } \\
\text { (postes ruraux) }\end{array}$ & 1,4 & 1,8 & 28 \\
\hline
\end{tabular}

Sources: Foreit, Vernon et Riveros 2005; León et al. 1998; Das et al. 2005; Sanogo et al. 2005; Vernon et al. 2005.

\section{Choisir uniquement les éta- blissements qui se prêtent bien à l'identification}

Tous les points de service ne conviennent pas à l'identification systématique. Celui-ci est surtout efficace dans les centres sous-utilisés où il est possible d'accroître la productivité. Par ailleurs, des centres qui fonctionnent à leur pleine capacité ne pourront pas mettre en ouvre cette stratégie sans augmenter le temps des prestataires, les ressources ou le temps d'attente des clientes.

L'identification systématique peut être mis en œuvre dans des établissements qui :

- Fournissent plus d'un service de santé à la fois.

- Disposent de prestataires qualifiés pour fournir les services demandés par les clientes lors de l'identification ainsi que des fournitures nécessaires pour dispenser ces services.

- Ne sont pas débordés avec un trop grand nombre de clients ou ne refusent pas les clients qui cherchent à obtenir des services.

- Ont des périodes creuses prévisibles. Souvent les centres sont très occupés à certains moments de la journée, mais pas à d'autres. Dans ce cas, vous pouvez décider d'utiliser l'identification systématique lorsqu'il y a moins de clients qui attendent pour les services.

\section{Observer la circulation des patientes avant de mettre en place le système d'identification et décider de la manière dont se déroulera cette identification}

Il est important de tracer le parcours qu'emprunteront les clientes dans l'établissement, puis de décider qui sera dépisté, quand cela sera effectué et comment on procédera.

Petits centres/postes: Souvent, il n'existe qu'un seul prestataire dans les petits centres et postes de santé. Ce prestataire est le seul point de contact des clientes et devrait réaliser tout l'identification. 
Etablissements plus grands: Généralement, il existe des prestataires différents pour tel ou tel type de service. Le premier prestataire que rencontrera la cliente pourrait être chargé de l'identification et devra ensuite orienter la cliente vers d'autres prestataires. Aussi, tous les prestataires de premier contact devront recevoir une formation portant sur l'utilisation de l'outil d'identification. On peut également décider que les clientes seront dépistées par le préposé à l'accueil s'il n'y pas trop de monde et s'il existe un endroit privé pour l'entretien. Après l'identification par le préposé à l'accueil, la cliente reçoit un coupon indiquant les services nécessaires et est informée de l'endroit où se trouve le prestataire qu'elle doit voir.

\section{Concevoir une nouvelle fiche ou adapter un outil d'identification exis- tant pour déterminer les services nécessaires}

L'outil d'identification est une fiche utilisée pour identifier les services dont peut avoir besoin une cliente. Deux exemples d'outils d'identification sont montrés ci-après. Certains programmes optent pour des fiches en papier pour obtenir l'information sur les clientes. La cliente va d'un prestataire à l'autre avec la fiche où sont notés les besoins dépistés et les services fournis. L'avantage de ces fiches en papier, c'est qu'elles peuvent être utilisées pour les orientations internes. L'inconvénient se situe au niveau des ruptures de stock de fiche. D'autres programmes prennent une approche moins chère et utilisent des fiches faites en plastique ou carton solide utilisées de nombreuses fois par le prestataire. L'inconvénient de cette approche, c'est qu'il est plus difficile de suivre le niveau d'identification effectué puisque l'outil reste avec le prestataire.
Quel que soit le type de fiche utilisée, elle doit :

Etre utilisée pour toutes les clientes en fonction de critères déterminés à l'avance et utilisé de la manière manière pour toutes les clientes.

- Dépister uniquement quelques besoins non satisfaits.

- Inclure des instructions de l'identification.

- Identifier le service pour lequel la cliente est venue avant l'identification d'autres besoins non satisfaits.

- Utiliser uniquement des réponses oui ou non.

- Faire la liste des services pour lesquels les clientes sont dépistées.

- Etre suffisamment courte et facile pour permettre aux prestataires de faire une identification en cinq minutes. Une fois qu'il aura plus d'expérience, le prestataire devrait y arriver en moins de temps.

La Figure 1 est une fiche qui avait l'objet d'un test réussi en Inde et au Sénégal. Cette fiche comprend chaque question qui doit être posée et la séquence dans laquelle les questions sont posées. 
Figure 1.

Liste de vérification utilisée en Inde et au Sénégal

\begin{tabular}{|c|c|c|c|c|c|}
\hline \multicolumn{6}{|c|}{ Outil d'identification } \\
\hline \multicolumn{6}{|c|}{ Date d'aujourd'hui: } \\
\hline \multicolumn{2}{|c|}{$\begin{array}{l}\text { Quel âge avez- } \\
\text { vous? }\end{array}$} & \multicolumn{4}{|c|}{$\begin{array}{l}\text { Administrer la liste de vérification uniquement si la femme a entre } 15 \text { et } 44 \text { ans. Si elle n'a pas entre } 15 \text { et } 44 \\
\text { ans, la remercier et terminer l'entretien. }\end{array}$} \\
\hline \multicolumn{5}{|c|}{ A remplir par l'agent d'identification } & Prestataire \\
\hline \multicolumn{3}{|c|}{$\begin{array}{l}\text { Questions d'identification } \\
\text { Note : Soyez sûr d'inclure la raison de la visite } \\
\text { dans des services requis. }\end{array}$} & Questions de suivi & $\begin{array}{l}\text { Discuter et } \\
\text { encercler le(s) } \\
\text { service(s) } \\
\text { nécessaire(s) }\end{array}$ & $\begin{array}{l}\text { Service } \\
\text { (résultat) }\end{array}$ \\
\hline \multicolumn{3}{|c|}{$\begin{array}{l}\text { Quelle est la raison principale de la visite } \\
\text { d'aujourd'hui ? }\end{array}$} & \multicolumn{2}{|l|}{ Raison de la visite : } & $\begin{array}{l}\text { 1. Fourni } \\
\text { 2. Planifié } \\
\text { 3. Référence }\end{array}$ \\
\hline 1 & $\begin{array}{l}\text { Etes-vous } \\
\text { 1. Oui } \rightarrow \\
\text { 2. Non: alle }\end{array}$ & $\begin{array}{l}\text { nceinte? } \\
\text { à } 2\end{array}$ & $\begin{array}{l}\text { Etes-vous suivie en consultation préna- } \\
\text { tale? } \\
\text { 1. Non } \rightarrow \\
\text { 2. Oui: aller à } 5\end{array}$ & $\begin{array}{l}\text { Consultations } \\
\text { prénatales } \\
\text { et aller à } 5\end{array}$ & $\begin{array}{l}\text { 1. Fourni } \\
\text { 2. Planifié } \\
\text { 3. Référence }\end{array}$ \\
\hline 2 & $\begin{array}{l}\text { Souhaitez- } \\
\text { 1. Non } \rightarrow \\
\text { 2. Oui: alle }\end{array}$ & $\begin{array}{l}\text { ous tomber enceinte? } \\
\text { à } 4\end{array}$ & $\begin{array}{l}\text { Utilisez-vous une méthode contracep- } \\
\text { tive? } \\
\text { 1. Non } \rightarrow \\
\text { 2. Oui: aller à } 3\end{array}$ & $\begin{array}{l}\text { Planification fami- } \\
\text { liale } \\
\text { et aller à } 4\end{array}$ & $\begin{array}{l}\text { 1. Fourni } \\
\text { 2. Planifié } \\
\text { 3. Référence }\end{array}$ \\
\hline 3 & $\begin{array}{l}\text { Etes-vous } \\
\text { contracepti } \\
\text { 1. Non } \rightarrow \\
\text { 2. Oui: alle }\end{array}$ & $\begin{array}{l}\text { atisfaite de votre méthode } \\
\text { è } \\
\text { à } 4\end{array}$ & $\begin{array}{l}\text { Aimeriez-vous utiliser une autre } \\
\text { méthode contraceptive? } \\
\text { 1. Oui } \rightarrow \\
\text { 2. Non: aller à } 4\end{array}$ & $\begin{array}{l}\text { Planification fami- } \\
\text { liale } \\
\text { et aller à } 4\end{array}$ & $\begin{array}{l}\text { 1. Fourni } \\
\text { 2. Planifié } \\
\text { 3. Référence }\end{array}$ \\
\hline 4 & $\begin{array}{l}\text { Quand ave } \\
\text { pour cance } \\
\text { 1. NSP/plu } \\
\text { 2. Moins de }\end{array}$ & $\begin{array}{l}\text {-vous eu votre dernier frottis } \\
\text { du col ? } \\
\text { de } 3 \text { ans } \rightarrow \\
3 \text { ans : aller à } 5\end{array}$ & $\begin{array}{l}\text { Souhaitez-vous un frottis cervical } \\
\text { aujourd'hui ? } \\
\text { 1. Oui } \rightarrow \\
\text { 2. Non: aller à } 5\end{array}$ & $\begin{array}{l}\text { Frottis } \\
\text { et aller à } 5\end{array}$ & $\begin{array}{l}\text { 1. Fourni } \\
\text { 2. Planifié } \\
\text { 3. Référence }\end{array}$ \\
\hline 5 & $\begin{array}{l}\text { Est-ce que } \\
\text { moins de } 5 \\
\text { 1. Oui } \rightarrow \\
\text { 2. Non: alle }\end{array}$ & $\begin{array}{l}\text { ous avez des enfants de } \\
\text { ns? } \\
\text { à } 7\end{array}$ & $\begin{array}{l}\text { Bénéficient-ils d'une consultation régu- } \\
\text { lière pour le suivi de leur croissante? } \\
\text { 1. Non } \rightarrow \\
\text { 2. Oui: aller à } 6\end{array}$ & $\begin{array}{l}\text { Evaluation de la } \\
\text { croissance et du } \\
\text { développement } \\
\text { et aller à } 6\end{array}$ & $\begin{array}{l}\text { 1. Fourni } \\
\text { 2. Planifié } \\
\text { 3. Référence }\end{array}$ \\
\hline 6 & $\begin{array}{l}\text { Tous vos e } \\
\text { complètem } \\
\text { 1. Non/NS } \\
\text { 2. Oui: alle }\end{array}$ & $\begin{array}{l}\text { ants de moins de } 5 \text { ans ont-ils } \\
\text { nt vaccinés? } \\
\rightarrow \\
\text { à } 7\end{array}$ & $\begin{array}{l}\text { Aimeriez-vous planifier leurs vaccina- } \\
\text { tions? } \\
\text { 1. Oui } \rightarrow \\
\text { 2. Non: aller à } 7\end{array}$ & $\begin{array}{l}\text { Vaccination } \\
\text { et aller à } 7\end{array}$ & $\begin{array}{l}\text { 1. Fourni } \\
\text { 2. Planifié } \\
\text { 3. Référence }\end{array}$ \\
\hline 7 & $\begin{array}{l}\text { Y a-t-il d'au } \\
\text { riez recevo } \\
\text { 1. Oui } \rightarrow \\
\text { 2. Non: Fin }\end{array}$ & $\begin{array}{l}\text { es services que vous aime- } \\
\text { aujourd'hui ou un autre jour? } \\
\text { le l'entretien }\end{array}$ & Liste de service(s) & & $\begin{array}{l}\text { 1. Fourni } \\
\text { 2. Planifié } \\
\text { 3. Référence }\end{array}$ \\
\hline \multicolumn{6}{|c|}{ Observations (agent d'identification) } \\
\hline \multicolumn{6}{|c|}{ Observations (prestataire): } \\
\hline & & fiche, l'attacher au do: & clinique de la clien & 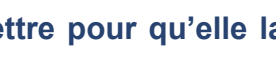 & 0 \\
\hline
\end{tabular}


Cet outil comprend cinq colonnes et sept rangées. L'agent d'identification remplit les questions dans les colonnes deux à quatre, et la dernière colonne sur le service (résultat) est remplie par le prestataire. La fiche doit être attachée au dossier clinique de la cliente après l'identification pour que le prestataire puisse remplir la fiche (en utilisant une version plastique ou en carton de la fiche).

Les agents d'identification doivent suivre une formation adéquate portant sur l'utilisation de l'outil. Suivant la réponse donnée aux questions dans les colonnes deux et trois, l'agent d'identification devra sauter certaines questions. Par exemple, dans la question 2, si la femme répond " oui » indiquant qu'elle souhaite tomber enceinte, l'agent d'identification doit passer à la question 4. Par ailleurs, si la femme répond "non, » - elle ne souhaite pas tomber enceinte - l'agent d'identification passe à la colonne suivante dans la même rangée et demande "Utilisezvous une méthode contraceptive ? "Si la réponse est " non, » l'agent d'identification passe à la colonne quatre et encercle la planification familiale et ensuite, passe à la question 4. Sauter ainsi d'une question à l'autre est probablement la tâche la plus difficile. Il est important que celui qui remplit la fiche d'identification comprenne bien ce mode correct de passer d'une question à l'autre.

Après avoir posé toutes les questions, l'agent d'identification passe à la colonne quatre et discute des services que souhaite recevoir la cliente. Si celle-ci souhaite recevoir des services pendant la même visite, le prestataire ou agent d'identification dispense les services et encercle le service « fourni » dans la dernière colonne. Si l'un ou plusieurs de ces services ne peuvent pas être fournis pendant la première consultation, le prestataire attache l'outil au dossier clinique et envoie la cliente avec ce dossier vers un autre prestataire. Si l'agent chargé de l'identification n'est pas le prestataire de soins, il oriente la cliente vers le prestataire qui traitera le problème.

La fiche d'identification peut être adaptée pour refléter les conditions et besoins locaux. University Research Co., LLC a adapté la fiche afin d'inclure les services concernant le VIH, notamment les conseils et l'identification volontaires (CDV). Il note également que la cliente ne voudra pas toujours accepter les services de planification familiale ou de conseils et identification volontaires du VIH lors de cette visite en particulier, mais qu'elle acceptera pourtant un dépliant d'information (voir Figure 2).

La fiche d'identification doit être évaluée après une certaine période d'utilisation pour déterminer si elle est toujours pertinente. Si telle question ne permet pas d'identifier les besoins non satisfaits pour ce service, elle sera éliminée de la fiche et remplacée par une autre question qui permet de mieux cerner les besoins actuels.

Figure 2.

\section{Questions de l'outil adapté de l'identification systématique}

\begin{tabular}{|c|c|c|c|c|}
\hline 2 & $\begin{array}{l}\text { Est-ce que vous souhaitez tomber } \\
\text { enceinte? } \\
\text { 1. Non } \rightarrow \\
\text { 2. Oui: aller à } 4\end{array}$ & $\begin{array}{l}\text { Est-ce que vous utilisez une méthode } \\
\text { contraceptive? } \\
\text { 1. Non } \rightarrow \text { et aller à } 4 \\
\text { 2. Oui: aller à } 3\end{array}$ & $\begin{array}{l}\text { Planification fami- } \\
\text { liale } \\
\text { Acceptée } \\
\text { Non acceptée, } \\
\text { dépliant remis }\end{array}$ & $\begin{array}{l}\text { 1. Fourni } \\
\text { 2. Planifié } \\
\text { 3. Référence }\end{array}$ \\
\hline 5 & $\begin{array}{l}\text { Avez-vous passé le test d'identifica- } \\
\text { tion du VIH? } \\
\text { 1. Non } \rightarrow \\
\text { 2. Oui: aller à } 6\end{array}$ & $\begin{array}{l}\text { Aimeriez-vous passer le test de } \\
\text { l'identification aujourd'hui ? } \\
\text { 1. Oui } \rightarrow \\
\text { 2. Non : aller à } 8\end{array}$ & $\begin{array}{l}\text { CDV/PTMAE } \\
\text { Acceptée } \\
\text { Non acceptée, } \\
\text { dépliant remis }\end{array}$ & $\begin{array}{l}\text { 1. Fourni } \\
\text { 2. Planifié } \\
\text { 3. Référence }\end{array}$ \\
\hline
\end{tabular}

Source: University Research Co., LLC 2006 adapté de Foreit 2006. 


\section{Prétest de la fiche}

La seule manière de savoir si la fiche est pratique et utile, c'est d'en faire le prétest. Il est parfois difficile pour certains agents de l'identification peu instruits ou débordés de travail d'administrer cette fiche. Et, par conséquent, on conseille de faire le prétest de deux types de fiches et de décider de celle qui convient le mieux dans le contexte donné. Il faut demander à trois prestataires au moins d'utiliser chaque outil avec au moins 10 clientes et de noter le temps qu'ils ont pris pour poser des questions. $\mathrm{Si}$ c'est possible, on observera l'identification. Peut-être faudra-t-il modifier la fiche si certaines questions sont difficiles à poser, si le mode pour sauter les questions n'est pas suivi correctement, si les questions ne sont pas comprises par la cliente ou s'il faut trop longtemps pour poser les questions de la fiche.

\section{Note!}

Lors du prétest de la fiche avec les clientes, il est obligatoire de fournir à chaque cliente les services qu'elle a demandés, soit le même jour, soit en lui donnant un rendez-vous pour un autre jour ou en l'envoyant vers d'autres services.

\section{Créer un manuel de l'utilisateur}

Une fois la fiche prétestée et finalisée, il faut rédiger un bref manuel de l'utilisateur qui explique la manière correcte d'administrer l'outil dans un langage clair pouvant être compris par les agents de l'identification.

\section{Formation adéquate}

Avant la formation, il est utile d'organiser une réunion avec tout le personnel de l'établissement pour expliquer les changements dans leur travail et dans le rôle que chacun devra assumer. Ecoutez-les et répondez à leurs préoccupations et demandez-leur leur avis sur la meilleure manière d'adopter la nouvelle straté- gie.

Lidentification systématique est une méthode très simple qui peut être enseignée en l'espace de quelques heures. Par ailleurs, nous ne recommandons pas de limiter la formation au minimum nécessaire. Nous avons constaté que, lorsque la période de formation est très courte, les participants ont tendance à ne pas prendre au sérieux l'identification et à ne pas l'adopter avec leurs clientes. La formation devrait durer au moins une journée, de préférence deux, et traiter les thèmes suivants :

- L'importance de l'identification.

- Les services mis en avant par l'identification.

- L'utilisation de l'outil (y compris le jeu de rôle ou des exercices pratiques dans un contexte clinique).

- Les droits de la cliente lors du processus d'identification (voir encadré ci-après).

- La tenue de dossiers et les comptes rendus.

- Comment prévoir une autre visite ou organiser une référence.

D’après notre expérience, en organisant une seconde journée de formation, on rend l'intervention de l'identification systématique plus efficace car les participants s'en souviennent davantage et les bonnes techniques d'identification sont renforcées grâce aux exercices pratiques. Nous recommandons que la seconde journée soit consacrée à des exercices pratiques supervisés avec des clientes de l'établissement dans les propres services du personnel. A la fin de la journée, les prestataires seront réunis pour discuter des besoins dépistés, des réponses des clientes à l'offre de services supplémentaires ou de toute autre question que peuvent avoir les prestataires à propos de la technique.

La formation sera d'autant plus efficace si un cadre supérieur de l'organisation la présente. En effet, sa présence montre que l'identification systématique est une intervention importante qui doit être utilisée par tous les participants à la formation.

Les superviseurs ont également besoin d'une formation. Ils devraient être formés lors des mêmes sessions que les personnes auxquelles on demandera de faire l'identification. Par ailleurs, plus d'une personne 


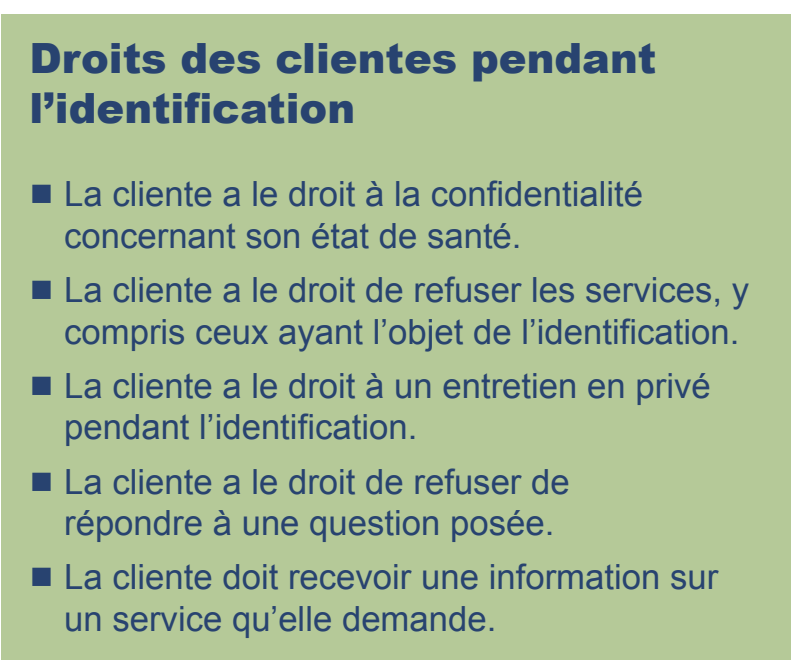

doit être formée par établissement. La cliente peut consulter l'un de plusieurs prestataires lors de la première visite (et identification), le personnel peut être malade, en vacance, être transféré ou avoir quitté le poste. Il faut avoir un remplaçant si l'agent ordinaire de l'identification ne peut pas venir au travail.

L'Annexe présente les grandes lignes d'un programme de formation.

\section{Supervision et suivi de l’identifica- tion}

Souvent, des outils et de nouvelles méthodes, à l'exemple de l'identification systématique, ne sont pas utilisés par les prestataires à cause d'un manque de supervision et de suivi. Si chaque établissement dispose d'un responsable de l'identification, la supervision sera meilleure. En plus, certaines informations sont collectées par le système régulier des statistiques de service sur le nombre de personnes dépistées. La collecte de données exigera peut-être une fiche supplémentaire ou de préférence une autre colonne dans les registres routiniers des prestataires ou de l'établissement. Si le programme comprend des comités de santé communautaires, ils doivent participer à la promotion et au suivi de l'intervention.
C'est surtout lors des premiers mois qu'il est important que le superviseur vérifie que les clientes sont effectivement dépistées et que tous les agents d'identification et prestataires utilisent la fiche et notent correctement les services fournis. Par la suite, le superviseur peut réaliser périodiquement une évaluation rapide en passant toute une journée dan le centre, en observant les services et en interviewant les clientes pour vérifier qu'elles ont effectivement bénéficié de l'identification. Le même jour, le superviseur discutera de chaque observation avec le prestataire observé, passant en revue les lacunes et félicitant le prestataire pour un travail bien fait.

Il est important de réévaluer la fiche d'identification après une certaine période d'utilisation. Si telle question ne permet pas d'identifier les besoins non satisfaits pour ce service, elle sera éliminée de la fiche et remplacée par une autre question qui permet de mieux cerner le besoin non satisfait pour d'autres services. 


\section{Références}

Brambila, C. and J. Solórzano. 1998. "Costos de las consultas de atención integral en salud reproductiva en Guatemala," INOPAL III Working Paper No. 7. New York: Population Council.

Das, N. P. et al. 2005. "Systematic screening to meet unmet need by integrating reproductive health services: An operations research model to maximize service utilization," FRONTIERS Final Report. Washington, DC: Population Council.

Foreit, Karen G. Fleischman, Karen Hardee, and Kokila Agarwal. 2002. "When does it make sense to consider integrating STI and HIV services with family planning services?" International Family Planning Perspectives 28(2): 105-107.

Foreit, James R., Ricardo Vernon, and Patricia Riveros. 2005. "Use of systematic screening to increase the provision of reproductive health services in Bolivia," FRONTIERS Final Report. Washington, DC: Population Council.
Foreit, James R. 2003. "FRONTIERS operations research protocols: Providing more preventive reproductive health care through systematic screening," FRONTIERS Report. Washington, DC: Population Council.

León, Federico et al. 1998. "Increasing use of reproductive health services in a Peruvian clinic," in J. Foreit and T. Frejka (eds.), Family Planning Operations Research: A Book of Readings. New York: Population Council, 239-245.

Sanogo, D. et al. 2005. "Using systematic screening to increase integration of reproductive health services delivery in Senegal," FRONTIERS Final Report. Washington, DC: Population Council.

Vernon, Ricardo et al. 2005. "Systematic screening as a strategy to increase services integration and revenues in Honduras," FRONTIERS Final Report. Washington, DC: Population Council.

Vernon, Ricardo and James Foreit. 1999. "How to help clients obtain more preventive reproductive health care," International Family Planning Perspectives 25(4): 200-202.

\section{Prière de consulter le site Web de FRONTIERS pour cette publication et d'autres :} http://www.popcouncil.org/frontiers 
Annexe

\section{Exemple de plan de formation pour les prestataires et leurs superviseurs}

Ce plan de formation de destine aux agents de l'identification, aux prestataires de soins et à leurs superviseurs. Il précise les résultats souhaités de la formation du point de vue connaissance et compétences (objectifs de formation), un exemple de programme de formation, un exemple de fiche d'identification et un exemple de jeu de rôle. On ne saurait souligner assez l'importance de la répétition pour la maîtrise de toute nouvelle compétence ou méthodologie. L'identification systématique réussi dépend de la compétence d'un prestataire à utiliser l'outil d'identification, aussi l'exercice est-il une priorité lors de toute formation.

Objectifs de la formation: Impartir les connaissances et les compétences dont auront besoin les prestataires et les superviseurs pour appliquer l'identification systématique dans leurs services :

- Avantages de l'identification systématique.

- Information sur chaque service ou programme dans le centre dans lequel ils travaillent : quand sont-ils disponibles (jours de semaine, heures), quels sont les prestataires pour chaque service, quels sont les services en train d'être intégrés à cette nouvelle intervention et, si le centre est payé pour ces services, quels sont les tarifs pour chaque service dispensé. De plus, les prestataires doivent savoir quels sont les services disponibles pour un rendez-vous ultérieur ou quels sont les services pour lesquels il faudra orienter la cliente vers un autre service ou une autre organisation.

- Questions éthiques/déontologiques : droits de la cliente lors de l'identification, notamment respecter le choix de la cliente d'être dépistée et de refuser un service que le prestataire juge nécessaire. Il est essentiel d'assurer le caractère privé et confidentiel de l'information donnée par la cliente.

- Connaissance de la fiche d'identification et être capable de l'utiliser — son objectif, la séquence des questions et les modes pour sauter les questions.
- Compétences pour utiliser la fiche d'identification telle qu'elle est conçue et pour la remplir avec toute l'information nécessaire.

- Tenue de dossiers et comptes rendus.

Il faut également indiquer aux prestataires qu'ils ne sont pas responsables de fournir tous les services euxmêmes, à moins qu'il n'y ait évidemment qu'un seul prestataire sur place. Ils sont responsables de faire l'identification des besoins non satisfaits des clientes, de les inscrire sur une fiche d'identification et de donner l'information nécessaire sur les services à la cliente.

La réussite de l'intervention dépend de l'utilisation régulière, exacte et constante de l'outil d'identification avec toutes les femmes en âge de procréer et, par conséquent, avant de démarrer l'intervention, il est important de vérifier que le prestataire dispose de la compétence nécessaire concernant les objectifs de formation susmentionnés. Dans certains cas, les prestataires auront besoin d'une pratique supervisée supplémentaire et d'un temps plus long pour achever la formation.

La formation devrait durer au moins un jour, de préférence deux. Tel que mentionné ci-dessus, d'après l'expérience, le second jour de la formation rend plus efficace l'intervention d'identification systématique puisque les participants s'en souviendront mieux et que les bonnes techniques d'identification sont renforcées par la répétition et la pratique. Nous recommandons que le second jour soit consacré à la pratique supervisée avec les clientes sur les lieux du travail du prestataire.

Pour éviter que ne soit interrompu l'identification, il faudrait former si possible au moins deux prestataires ou deux membres du personnel clinique par établissement, plus les superviseurs.

Exemple de programme de formation pour les prestataires et leurs superviseurs. 


\section{Premier jour}

Lors du matin du premier jour, on s'assure que le personnel connaît bien l'intervention, les services dispensés dans les centres qui participent et de la fiche d'identification en question. Il faut revoir et expliquer son utilisation, la séquence des questions et les modes pour sauter les questions jusqu'à ce que tous les participants aient bien compris leur application.

L'après-midi du premier jour, le personnel s'exercera à utiliser l'outil d'identification dans le cadre de trois jeux de rôle au minimum. Il est important de passer en revue les droits de la cliente lors de l'identification :

- La cliente a le droit à la confidentialité.

- La cliente a le droit de refuser les services.

- La cliente a le droit à un entretien en privé pendant l'identification.

- La cliente a le droit de refuser de répondre à une question posée.

- La cliente doit recevoir une information sur un service qu'elle demande.

Un exemple de jeu de rôle est donné ci-après. Le formateur devra organiser d'autres jeux de rôle en utilisant des profils de clientes typiques qui viennent dans ce centre avec les besoins de services spécifiques choisis aux fins d'intégration. Les profils dans les cas permettront aux prestataires de recevoir une formation pour s'exercer à poser les questions dans la bonne séquence et à utiliser correctement les modes pour sauter les questions. Le ou les superviseur(s) doivent également assister à la séance entière et être formés pour savoir utiliser la fiche d'identification, de pair avec le personnel clinique.

\section{Exemple d'exercice de jeu de rôle pour l'identification systématique}

1. Divisez le personne en groupes de trois personnes; l'une sera l'agent d'identification, l'autre la cliente et l'autre l'évaluateur.

2. Donnez son profil à la « cliente » (voir exemples ci-après) et donnez-lui le temps de l'étudier pendant quelques minutes. Dites-lui qu'elle ne devra

\section{Exemple de profil d'une cliente utilisé pour le jeu de rôle de la cliente \\ - La cliente a 27 ans et elle est en bonnesanté. \\ - Elle vient au centre aujourd'hui avec sa fillette de 9 mois car celle-ci doit recevoir son vaccin contre la rougeole et la rubéole. \\ - Elle n'est pas enceinte (elle a eu ses règles il y a 2 semaines) et ne souhaite pas tomber enceinte. \\ - Elle n'utilise pas de contraception. \\ - Son dernier frottis remonte à un an. \\ - Elle a un autre enfant, un garçon de 3 ans, qui est venu avec elle aujourd'hui. \\ - Aucun suivi de croissance n'a été donné à son garçon car personne n'a offert ce service. \\ - Son fils a reçu toutes les vaccinations néces- saires.}

indiquer à l'agent d'identification/prestataire que le service motivant sa visite. Le reste de l'histoire devra être donné petit à petit si l'agent d'identification demande cette information.

3. Remettre à "l'agent d'identification " un outil d'identification en blanc et demandez-lui de faire l'identification et de remplir la fiche.

4. "L'évaluateur " reçoit une fiche d'identification avec les étapes correctes en fonction du profil de la cliente, des questions de suivi et des questions à sauter et avec les besoins en services notés sur la fiche (voir la seconde fiche d'identification ciaprès). Il soulignera les étapes qui n'ont pas été effectuées correctement.

5. A la fin du jeu de rôle, le formateur rencontrera les trois membres du personnel, évaluera leur performance et corrigera toute mauvaise compréhension ou autre lacune.

6. Par la suite, dans le même trio, les rôles seront changés avec un nouveau profil de cliente.

Essayez de le réaliser au moins trois fois pour chaque membre ait l'occasion d'évaluer une identification, d'être une cliente et un agent d'identification. 
Notes sur la fiche d'identification: Chaque question reçoit une réponse « oui » ou «non » et la fiche dirige l'agent d'identification vers une nouvelle question ou le service devant être fourni suivant un mode indiqué par flèche. Par exemple, à la question 2, si la femme répond " oui » à la question lui demandant si elle souhaite tomber enceinte, l'agent d'identification passe à la question 4. Par ailleurs, si la femme répond " non » à la question lui demandant si elle souhaite tomber enceinte, l'agent d'identification reste dans la seconde rangée et demande " Est-ce que vous utilisez un contraceptif ? " Si la réponse est "non, » il passe à la colonne quatre et encercle Planification familiale et ensuite, saute à la question 4. Après avoir répondu à la série de questions, l'agent d'identification revient à la colonne quatre et discute des services que souhaite la cliente. Si celle-ci souhaite recevoir le service, l'agent d'identification attache l'outil au dossier clinique et le transmet au prestataire. Le prestataire encercle le service approprié (" fourni, " " planifié " ou « référence») dans la dernière colonne. La dernière colonne peut être éliminée si que la fiche reste avec le prestataire. Dans ce cas, dans les centres avec plusieurs bureaux, les besoins d'orientation interne doivent se faire d'une autre manière, probablement oralement. 


\section{Outil d'identification (à utiliser par l'agent d'identification lors du jeu de rôle)}

Date d'aujourd'hui:

Quel âge avez- $\quad$ Administrer la liste de vérification uniquement si la femme a entre 15 et 44 ans. Si elle n'a pas entre 15 et 44 vous? ans, la remercier et terminer l'entretien.

\section{A remplir par l'agent d'identification}

\section{Questions d'identification}

Note : Soyez sûr d'inclure la raison de la visite dans des services requis.

\section{Questions de suivi}

Quelle est la raison principale de la visite Raison de la visite :

d'aujourd'hui ?

Etes-vous enceinte ?
1. Oui $\rightarrow$
2. Non: aller à 2
$2 \quad$ Souhaitez-vous tomber enceinte?
1. Non $\rightarrow$
2. Oui: aller à 4

3 Etes-vous satisfaite de votre méthode contraceptive?

\section{Non $\rightarrow$}

2. Oui: aller à 4

4 Quand avez-vous eu votre dernier frottis pour cancer du col?

1. NSP/plus de 3 ans $\rightarrow$

2. Moins de 3 ans : aller à 5

$5 \quad$ Est-ce que vous avez des enfants de moins de 5 ans ?

\section{Oui $\rightarrow$}

2. Non: aller à 7

6 Tous vos enfants de moins de 5 ans ont-ils complètement vaccinés?

\section{Non/NSP $\rightarrow$}

2. Oui: aller à 7

$7 \quad$ Y a-t-il d'autres services que vous aime- $\quad$ Liste de service(s)

riez recevoir aujourd'hui ou un autre jour?

1. Oui $\rightarrow$

2. Non: Fin de l'entretien

Etes-vous suivie en consultation prénatale?

1. Non $\rightarrow$

2. Oui: aller à 5

Utilisez-vous une méthode contraceptive ?

1. Non $\rightarrow$

2. Oui: aller à 3

Aimeriez-vous utiliser une autre méthode contraceptive ?

1. Oui $\rightarrow$

2. Non: aller à 4

Souhaitez-vous un frottis cervical aujourd'hui ?

1. Oui $\rightarrow$

2. Non: aller à 5

Bénéficient-ils d'une consultation régulière pour le suivi de leur croissante?

1. Non $\rightarrow$

2. Oui: aller à 6

Aimeriez-vous planifier leurs vaccinations?

1. Oui $\rightarrow$

2. Non: aller à 7

Observations (agent d'identification) :
Prestataire

Discuter et
encercler le(s)
service(s)

nécessaire(s)

Service (résultat)

1. Fourni

2. Planifié

3. Référence

Consultations 1. Fourni prénatales

2. Planifié

et aller à 5

3. Référence

Planification fami- 1. Fourni

liale

et aller à 4

2. Planifié

3. Référence

Planification fami- 1. Fourni

liale 2. Planifié

et aller à $4 \quad 3$. Référence

Frottis

1. Fourni

et aller à 5

2. Planifié

3. Référence

Evaluation de la

1. Fourni croissance et du développement et aller à 6

2. Planifié

3. Référence

taccination

Vaccination

et aller à 7

1. Fourni

2. Planifié

3. Référence

1. Fourni

2. Planifié

3. Référence

Observations (prestataire) :

Après avoir rempli la fiche, l'attacher au dossier clinique de la cliente ou la lui remettre pour qu'elle la présente au prestataire de services. 


\section{Outil d’identification (à utiliser par l'évaluateur lors du jeu de role pour documenter une identification correct de la part du prestaire)}

Date d'aujourd'hui:

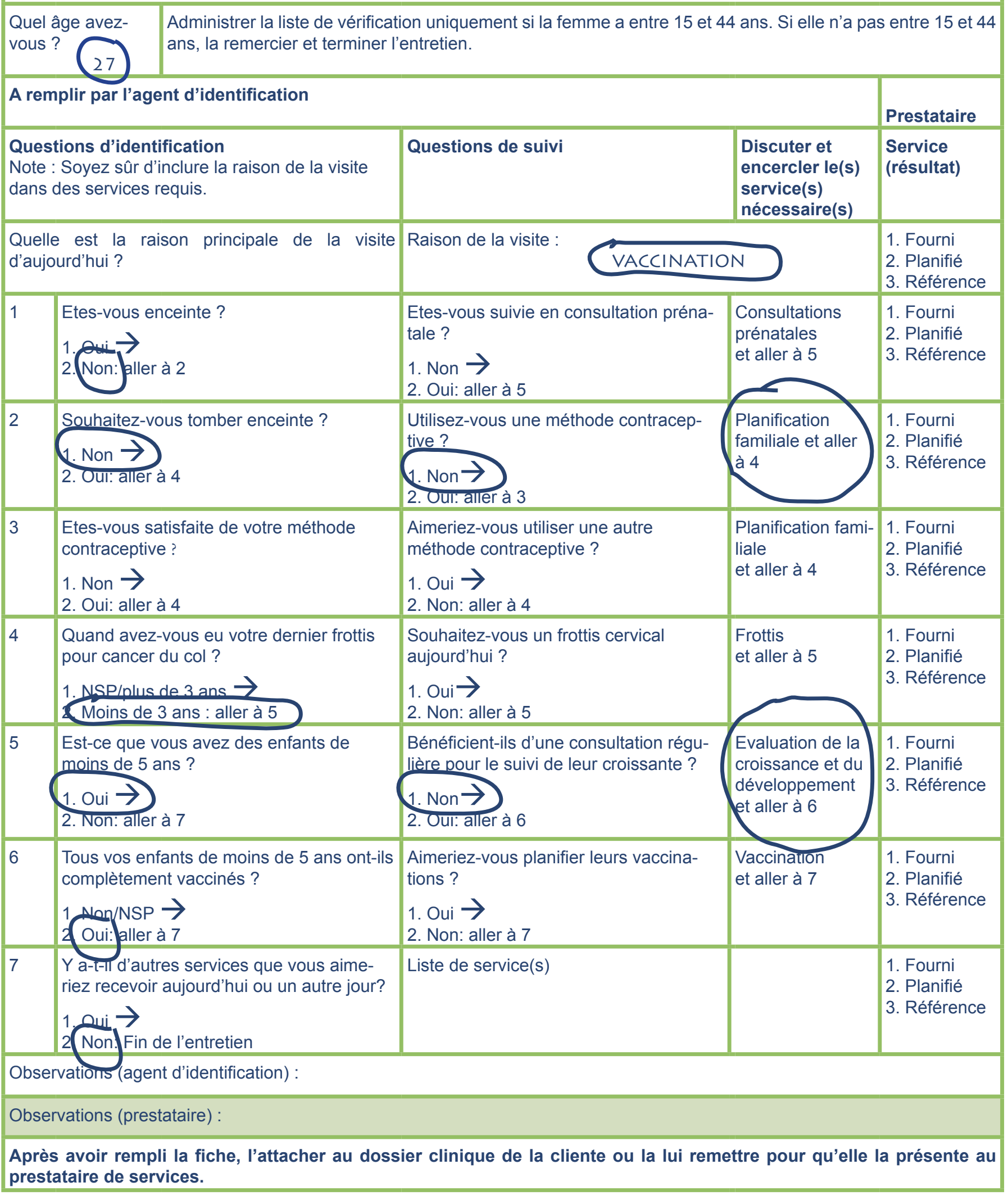

*Note : la quatrième colonne sur le résultat du service n'est pas remplie. Elle doit être remplie par le prestataire pour les dossiers. 


\section{Deuxième jour}

D'après l'expérience dans plusieurs pays, la seconde journée de formation permet une adoption plus efficace de l'intervention d'identification systématique puisque les bonnes techniques d'identification sont renforcées grâce à davantage de pratique.

La seconde journée est consacrée à une pratique supervisée avec des clientes de l'établissement sur les lieux du travail du personnel. On demande aux clientes de permettre au personnel de faire l'identification de leurs besoins en santé de la reproduction et santé infantile. Le personnel fera l'identification et remplira l'outil, puis le remettra au prestataire de soins. A présent, le superviseur assume son rôle de supervision, suit les questions du personnel et lui donne un feedback.

Il est important de comprendre qu'une fois qu'une cliente a reçu l'identification, même si ce n'est qu'une partie de la formation, il faut lui fournir tous les services selon les besoins identifiés. Les services doivent être dispensés à la cliente soit pendant la même visite, soit en lui donnant un rendez-vous pour une visite ultérieure dans le centre ou en l'envoyant vers un autre centre qui fournit le service nécessaire.
Le formateur doit prendre le temps nécessaire après chaque identification pour discuter de la performance des participants (prestataire et superviseur) pendant cette identification particulier pour aider à corriger toute lacune notée. Cette séquence sera répétée jusqu'à ce que le personnel et le superviseur maitrisent toutes les étapes de l'identification et de la prestation de services. Le formation sera responsable de certifier que le personnel est compétent pour assumer l'identification des clientes lors de la prestation de services. Le personnel devrait dépister un minimum de cinq clientes ou de clientes simulées pour être jugé compétent.

En guise de conclusion, les formateurs motiveront encore davantage le prestataire face à cette identification grâce à une discussion de groupe sur leurs expériences, notamment :

- Le nombre de besoins non satisfaits dépistés.

- Les réponses des femmes à l'offre de services complémentaires.

- Tout problème rencontré et toute solution éventuelle. 


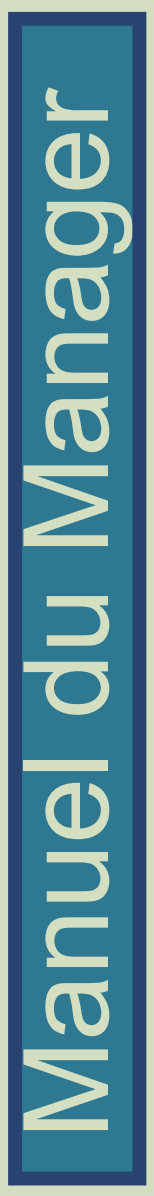

